\title{
Pulmonary venous occlusion and death in pulmonary arterial hypertension: survival analyses using radiographic surrogates
}

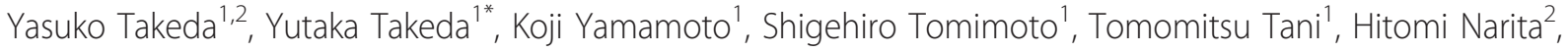
Nobuyuki Ohte ${ }^{1}$ and Genjiro Kimura ${ }^{1}$

\begin{abstract}
Background: Recent studies find that a considerable number of patients with pulmonary arterial hypertension (PAH) develop fibrous obstruction of the pulmonary veins. Such obstruction more commonly accompanies connective tissue disorder (CTD)-associated PAH than idiopathic PAH. However, few researchers have gauged the risk of death involving obstruction of the pulmonary veins.

Methods: Thirty-seven patients with PAH were enrolled (18 patients, idiopathic PAH; 19 patients, CTD-associated PAH). The patients were $49 \pm 18$ years and had a World Health Organization functional class of $3.2 \pm 0.6$. Thickening of the interlobular septa, centrilobular ground-glass attenuation, and mediastinal adenopathy were surrogates for obstruction of the pulmonary veins, and were detected by a 16-row multidetector computed tomography scanner.

Results: The follow-up period was $714 \pm 552$ days. Fifteen deaths occurred. Thickening of the interlobular septa, centrilobular ground-glass attenuation, and mediastinal adenopathy were found in 37.8\%, 24.3\%, and 16.2\% of patients, respectively. Cox proportional hazard analysis revealed an increased risk of death with each radiographic surrogate (mediastinal adenopathy: $p<0.0001$, hazard ratio $=13.9$; thickening of interlobular septa: $p<0.001$, hazard ratio $=12.0$; ground-glass attenuation: $p=0.02$, hazard ratio $=3.7$ ). The statistical significance of these relationships was independent of the cause of PAH and plasma concentration of brain natriuretic peptide.
\end{abstract}

Conclusions: The results of this study imply that obstruction of the pulmonary veins is associated with an increased risk of death in patients with PAH.

\section{Background}

Pulmonary arterial hypertension (PAH) carries a significant risk of death $[1,2]$. Patients with a poor response to pulmonary vasodilating agents often die within several years [1]. Recent studies show that a considerable number of patients with PAH develop fibrous obstruction in the pulmonary veins $[3,4]$. These findings challenge relationship between clinical entity of $\mathrm{PAH}$ and that of pulmonary veno-occlusive disease (PVOD). Case reports suggest that obstruction of pulmonary veins is associated with a poor prognosis [5-8]. However, few

\footnotetext{
* Correspondence: takeday@med.nagoya-cu.ac.jp 'Department of Cardio-Renal Medicine and Hypertension, Nagoya City University Graduate School of Medical Sciences, Nagoya, Japan Full list of author information is available at the end of the article
}

researchers have gauged the risk of death due to pulmonary venous obstruction. To assess the effect of pulmonary venous obstruction on the risk of death in patients with $\mathrm{PAH}$, this study performed survival analyses with patients grouped by the cause of PAH and by the presence or absence of radiographic surrogates for obstruction of the pulmonary veins.

\section{Methods}

The patients

Forty-five patients with idiopathic PAH or connective tissue disorder (CTD)-associated PAH were referred to our PAH clinic from January 2004 to March 2009. We excluded eight patients from analyses for the following reasons: a total lung capacity less than $70 \%$ of the 
predicted value (two patients); a serum creatinine concentration greater than $2.5 \mathrm{mg} / \mathrm{dL}$ (two patients); an age greater than 75 years (two patients); the presence of liver cirrhosis (one patient); and the presence of gastric cancer (one patient). The remaining 37 study participants (8 men and 29 women) gave informed consent. PAH was diagnosed from the results of right heart catheterisation at rest $[9,10]$. PAH was defined as a mean pulmonary artery pressure greater than $25 \mathrm{~mm}$ $\mathrm{Hg}$, a pulmonary capillary wedge pressure less than or equal to $15 \mathrm{~mm} \mathrm{Hg}$, and pulmonary vascular resistance greater than 3 Wood units. All of the patients completed the evaluations, as described below. However, the data of right heart catheterisation from 11 patients were not used for analyses for the change of the treatment between catheterisation and the other baseline evaluations. Pulmonary arterial thromboembolism was not detected in any of the patients with a lung perfusion scan. The institutional ethics committee approved the protocol. All the participants gave informed consent to this study.

\section{Assessment of radiographic surrogates of pulmonary venous obstruction}

Thickening of the interlobular septa, the presence of centrilobular ground-glass attenuation, and enlargement of the mediastinal lymph nodes were surrogates for obstruction of the pulmonary veins; these surrogates were detected with computed tomography (CT) scans (Figure 1) [5,7-9,11-16]. The details of these abnormalities are described elsewhere [15,17-19]. A 16-row multidetector CT scanner (IDT 16, Philips Electronic Japan Medical Systems, Tokyo, Japan) used the following scan

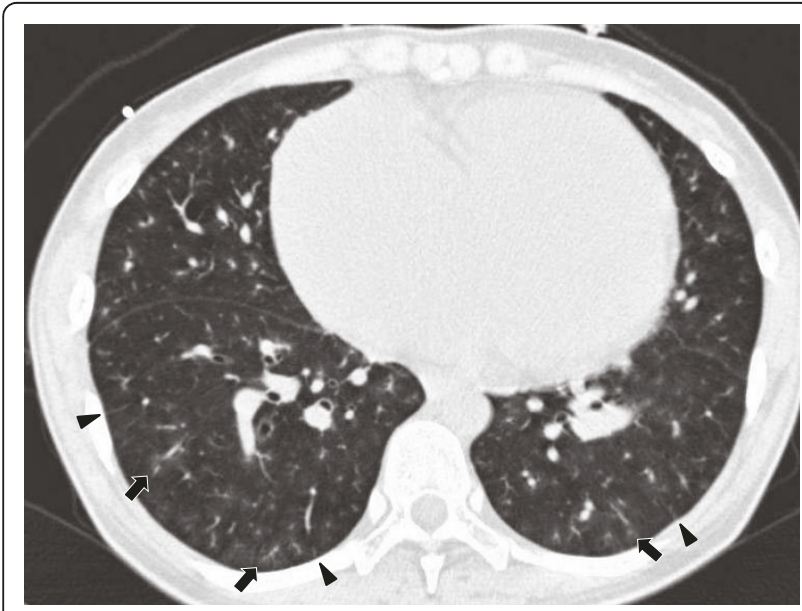

Figure 1 The computed tomography scan of a 25-year-old woman with severe idiopathic pulmonary arterial hypertension reveals thickening of the interlobular septa (arrowheads) and small multifocal areas of centrilobular ground-glass attenuation. parameters: simultaneous acquisition of 16 slices per rotation with a slice thickness of $0.75 \mathrm{~mm}$, rotation time of $500 \mathrm{~ms}$, pitch of $0.9 \mathrm{~mm}$, tube voltage of $120 \mathrm{kV}$, and tube current of $200 \mathrm{~mA}$. Cross-sectional images were reconstructed with a slice thickness of $3.0 \mathrm{~mm}$ at 3.0 $\mathrm{mm}$ intervals. Board-certified radiologists gave reports on the CT images within 24 hours after the scans. Our radiologists were not restricted from other clinical data. Two investigators assessed the CT images and the reports for the surrogates within a few days thereafter. Final decisions were reached by consensus if there was an inconsistency.

\section{Assessment of the pulmonary hypertension status}

One investigator assessed each patient's functional class in accordance with the World Health Organisation (WHO) criteria. The patients underwent an overnight fast. Blood samples were afterwards drawn from a peripheral vein. The samples were immediately placed on ice and centrifuged at $4^{\circ} \mathrm{C}$. The plasma concentration of brain natriuretic peptide (BNP) was then measured. An external laboratory (SRL Inc., Tokyo, Japan) measured BNP concentrations using an immunoradiometric assay. A trained sonographer recorded all echocardiograms using a commercially available system (SSA-770A, Toshiba Medical Co. Ltd, Tokyo, Japan). The cardiac index and Doppler right ventricular index were calculated using Doppler echo techniques [20]. Each measurement was the average of three or more consecutive beats at the end expiration.

\section{Follow-up}

The baseline was defined as the day of the CT scan. CT scan was taken just before initiation of treatment in out institute. The patients were asked to visit our outpatient clinic every four weeks to adjust medical therapy and to obtain information on clinical events. In addition to these periodical contacts, the investigators and the patients sometimes communicated by phone. (If a patient did not come, the investigators interviewed the patients or their family by phone.) The treatment was not controlled because of the generally high mortality. Treatment failure was defined as death, hospitalisation due to cardiovascular events (including syncope), use of inotropic agents, elevation of a patient's WHO functional class, or a three-fold increase in the plasma BNP concentration [21].

\section{Statistical analyses}

Statistical analyses were performed with the Statistical Package for Social Science version 15.0 for Windows (SPSS Inc., Chicago, Illinois). Survival times were defined as the length of time a patient lived from the baseline date to Dec 31, 2009. Data are expressed as the 
mean \pm the standard deviation. The plasma BNP concentrations were transformed to their natural logarithms to normalise the distribution, and the BNP data are expressed as the median with the 25th and 75th percentiles. Categorical variables were compared using the chisquare test. The effect of each radiographic surrogate for pulmonary venous obstruction and the effect of each cause of PAH in relation to the patients' characteristics were tested with a two-way analysis of variance. The prognostic values of the variables were tested using Cox proportional-hazards regression analyses. The results are expressed as the hazard ratios with $95 \%$ confidence intervals. The Kaplan-Meier method produced the survival curves. A two-sided $p$ value less than 0.05 was considered statistically significant.

\section{Results}

\section{Patients' characteristics and follow-up results}

Table 1 shows the characteristics of the patients. The follow-up period was $714 \pm 552$ days. All patients maintained their follow-up visits. Death occurred in 5 (24\%) class III patients and in 10 (83\%) class IV patients, but no deaths occurred in class II patients $(p<0.001)$. Death occurred in 9 (50\%) in patients with idiopathic PAH and in $6(32 \%)$ in patients with CTD-associated PAH ( $1=0.23)$. Heart failure was the cause of death in all but two mortality cases. One of these two patients died of septic shock and the other died of a massive

Table 1 Baseline characteristics of the patients

\begin{tabular}{ll}
\hline Variables & $\mathbf{n}=\mathbf{3 7}$ \\
\hline Age (yrs) & $49.0 \pm 18.1$ \\
Women & $29(78.4)$ \\
Time from the onset to study enrolment (weeks) & $14.2 \pm 18.3$ \\
Functional class II/III/IV & $4 / 21 / 12(10.8 / 56.8 /$ \\
& $32.4)$ \\
Idiopathic PAH/connective tissue disorder- & $18 / 19(48.6 / 51.4)$ \\
associated PAH & \\
Death & $15(40.5)$ \\
Treatment failure* & $20(54.1)$ \\
Use of PAH-specific drug at baseline & \\
Epoprostenol alone & $11(29.7)$ \\
Sildenafil alone & $4(10.8)$ \\
Bosentan alone & $6(16.2)$ \\
Beraprost alone & $13(35.1)$ \\
Epoprostenol + Sildenafil & $1(2.7)$ \\
Sildenafil + Beraprost & $1(2.7)$ \\
Sildenafil + Bosentan + Beraprost & $1(2.7)$ \\
Oxygen & $33(89.2)$ \\
\hline
\end{tabular}

Values are presented as the number (percent) or as the mean \pm standard deviation (SD).

*Treatment failure was defined as death, hospitalisation due to cardiovascular event (including syncope), use of inotropic agents, or elevation in a patient's WHO functional class.

Abbreviation: $\mathrm{PAH}$, pulmonary arterial hypertension. lung haemorrhage. Treatment failure occurred in 10 (83\%) class IV patients; nine (43\%) class III patients; and one $(20 \%)$ class II patient $(p=0.038)$. No patient received lung or heart-lung transplantation. All patients experienced an increase in the use of pulmonary vasodilating agents.

\section{Radiographic surrogates for obstruction of the pulmonary veins}

The frequency of each radiographic surrogate for pulmonary venous obstruction was shown in Table 2 . There was no statistical difference between the causes of $\mathrm{PAH}$ in the frequency of each surrogate (mediastinal adenopathy: $p=0.21$; thickening of interlobular septa: $p$ $=0.42$; ground-glass attenuation: $p=0.77$ ). The haemodynamic data and plasma concentration of BNP were comparable between the causes of PAH. Mediastinal adenopathy was associated with an advanced age and a high plasma concentration of BNP. Thickening of interlobular septa was associated with a low cardiac index and high plasma concentration of BNP. Centrilobular ground-glass attenuation was not associated with any of the haemodynamic data or with the plasma concentration of BNP.

Radiographic surrogates tended to appear with each other. Four patients had three surrogates; nine patients had two surrogates; two patients had one surrogate, and 22 patients had no surrogates.

\section{Survival rate and radiographic surrogates for obstruction} of the pulmonary veins

Survival analyses showed that each radiographic surrogate for pulmonary venous obstruction was associated with a seriously increased risk of death. Cox proportional hazard analysis using mediastinal adenopathy as a surrogate found that the adenopathy was associated with a seriously increased risk of death $(p<0.0001$; hazard ratio $[95 \% \mathrm{CI}])=13.9[4.2-47.6]$ ). Adding the cause of PAH to the covariate did not weaken the relationship between mediastinal adenopathy and the risk of death (adenopathy: $p<0.00001$, hazard ratio [95\% $\mathrm{CI}])=14.1$ [4.1 - 47.9]; causes of PAH: $p=0.26$ ); the survival curves of patients with each cause of PAH were close after being stratified by the presence or absence of mediastinal adenopathy (Figure 2). Given the relationship with an elevated plasma concentration of BNP, Cox proportional analysis was also performed by adding the plasma BNP concentration to the covariates. The analysis showed that the adenopathy was associated with a seriously increased mortality rate independently of the BNP concentration (adenopathy: $p=0.006$, hazard ratio $[95 \% \mathrm{CI}]=7.0[1.9-26.3]$; BNP: $p=0.013$, hazard ratio $[95 \% \mathrm{CI}]=2.0[1.2-3.4]$; the causes of PAH: $p=0.78$ ). 
Table 2 Characteristics of the patients with each sign of pulmonary venous obstruction

\begin{tabular}{|c|c|c|c|c|c|c|c|c|}
\hline Signs & & Cause & $\mathrm{n}$ & Age(years) & $\mathrm{mRAP}(\mathrm{mm} \mathrm{Hg})^{\S}$ & mPAP $(\mathrm{mm} \mathrm{Hg})^{\S}$ & $\mathrm{Cl}(\mathrm{L} / \mathrm{min} / \mathrm{m} 2)$ & $\mathrm{BNP}(\mathrm{pg} / \mathrm{mL})$ \\
\hline Mediastinal & Yes & Idiopathic & 6 & $56 \pm 18^{*}$, & $9 \pm 4$ & $43 \pm 5$ & $1.9 \pm 0.4$ & $860[637-1210]^{* *}$ \\
\hline \multirow[t]{3}{*}{ adenopathy } & & CTD & 3 & $65 \pm 3^{*}, \#$ & $8 \pm 3$ & $36 \pm 19$ & $2.5 \pm 0.3$ & $148[83-959]^{* *}$ \\
\hline & No & Idiopathic & 12 & $37 \pm 17^{*}$, & $8 \pm 3$ & $62 \pm 16$ & $2.4 \pm 0.7$ & $133[37-418]^{* *}$ \\
\hline & & $\overline{C T D}$ & 16 & $52 \pm 16^{* \#}$ & $7 \pm 4$ & $48 \pm 19$ & $2.5 \pm 0.9$ & $141[75-334]^{* *}$ \\
\hline Thickening of & Yes & Idiopathic & 8 & $50 \pm 19$ & $9 \pm 5$ & $56 \pm 16$ & $1.9 \pm 0.4^{*}$ & 800 [487-1062]* $^{*}$ \\
\hline \multirow[t]{3}{*}{ interlobular septa } & & CTD & 6 & $49 \pm 18$ & $7 \pm 4$ & $42 \pm 18$ & $2.0 \pm 0.4^{*}$ & 482 [83-959]* $^{*}$ \\
\hline & No & Idiopathic & 10 & $38 \pm 19$ & $7 \pm 2$ & $56 \pm 16$ & $2.5 \pm 0.7^{*}$ & $92[28-333]^{*}$ \\
\hline & & CTD & 13 & $56 \pm 14$ & $7 \pm 3$ & $42 \pm 18$ & $2.7 \pm 0.9^{*}$ & $126[34-290]^{*}$ \\
\hline Centrilobular & Yes & Idiopathic & 4 & $39 \pm 20$ & $9 \pm 5$ & $56 \pm 16$ & $2.1 \pm 0.5$ & $487[236-715]$ \\
\hline ground-glass & & CTD & 5 & $54 \pm 24$ & $9 \pm 4$ & $49 \pm 15$ & $2.0 \pm 0.5$ & 148 [141 - 959] \\
\hline \multirow[t]{2}{*}{ attenuation } & No & Idiopathic & 14 & $45 \pm 19$ & $8 \pm 3$ & $61 \pm 18$ & $2.3 \pm 0.7$ & $263[46-806]$ \\
\hline & & CTD & 14 & $55 \pm 13$ & $6 \pm 3$ & $45 \pm 20$ & $2.7 \pm 0.9$ & $111[34-334]$ \\
\hline
\end{tabular}

Values are number (percent) or mean \pm SD, as appropriate. Data of BNP are shown as median [25percentile, 75percentile]. $P$ values for the comparison between the patients with and without each radiographic surrogate for obstruction of the pulmonary veins; $*^{*}<0.02$ and ${ }^{* *}:<0.01$. $P$ values for the comparison for the difference between the causes; ${ }^{\#}:<0.03$. No mark means that there is no significant effect of either the presence and absence of each radiographic surrogate or the difference in the cause. ${ }^{\S}$ : The values for mRAP and mPAP are based on 26 patients. BNP = brain natriuretic peptide, $\mathrm{Cl}=\mathrm{cardiac}$ index, $\mathrm{CTD}=$ connective tissue disorder, $\mathrm{mRAP}=$ mean right atrial pressure, $\mathrm{mPAP}=$ mean pulmonary arterial pressure, $\mathrm{RV}=$ right ventricle, $\mathrm{SD}=\mathrm{standard}$ deviation .

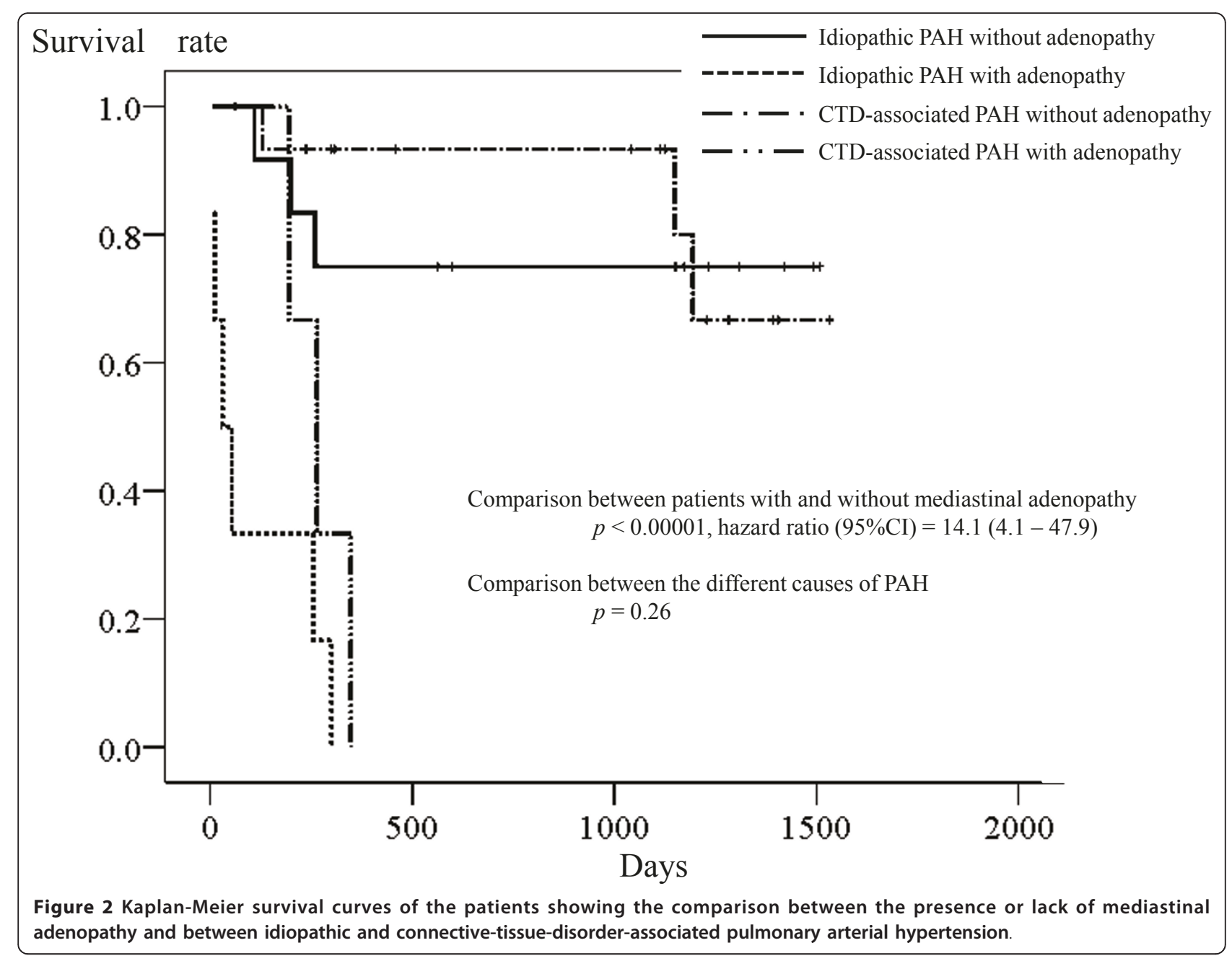


Cox proportional hazard analysis found that thickening of the interlobular septa was also associated with a seriously increased risk of death $(p<0.001$; hazard ratio $[95 \% \mathrm{CI}]=12.0[3.2-45.5])$. Adding the cause of $\mathrm{PAH}$ to the covariate had little impact on the relationship between this surrogate and the risk of death (thickening: $p<0.001$, hazard ratio $[95 \% \mathrm{CI}]=11.6[3.1-43.5]$; the causes of PAH: $p=0.29$ ); the survival curves of patients with each cause of PAH were, similar to the results of mediastinal adenopathy, close after stratification by the presence or absence of thickening of the interlobular septa (Figure 3). Given the relationship with an elevated plasma BNP concentration, Cox proportional analysis was also performed by adding the plasma BNP concentration to the covariates. The analysis again showed that interlobular septal thickening was associated with a seriously increased mortality rate independently of the concentration of BNP (thickening: $p=0.004$, hazard ratio $[95 \% \mathrm{CI}]=8.9[1.9-41.7] ; \mathrm{BNP}: \mathrm{p}=0.005$, hazard ratio $[95 \% \mathrm{CI}]=2.0[1.3-3.3]$; the causes of PAH: $p=0.30$ ).
The presence of centrilobular ground-glass attenuation was also associated with an increased risk of death, although the effect with this surrogate was somewhat milder than with the other surrogates $(p=0.02$, hazard ratio $[95 \% \mathrm{CI}]=3.7[1.2-11.1])$. Adding the causes of $\mathrm{PAH}$ to the covariate had little impact on the relationship between this surrogate and the risk of death (attenuation: $p=0.02$, hazard ratio $[95 \% \mathrm{CI}]=3.6[1.2$ 10.8]; the causes of PAH: $p=0.24$ ); the survival curves were somewhat close in patients with ground glass attenuation regardless the cause of $\mathrm{PAH}$, although this is less clear than with the other two surrogates (Figure 4). Despite the relationship between this surrogate and the elevation of plasma BNP level did not reach the statistical significance, Cox proportional analysis was performed by adding plasma concentration of BNP to the covariates. The analysis again showed that ground-glass attenuation was associated with a seriously increased mortality rate independently of the BNP concentration (attenuation: $p=0.016$, hazard ratio $[95 \% \mathrm{CI}]=4.8[1.3$ -

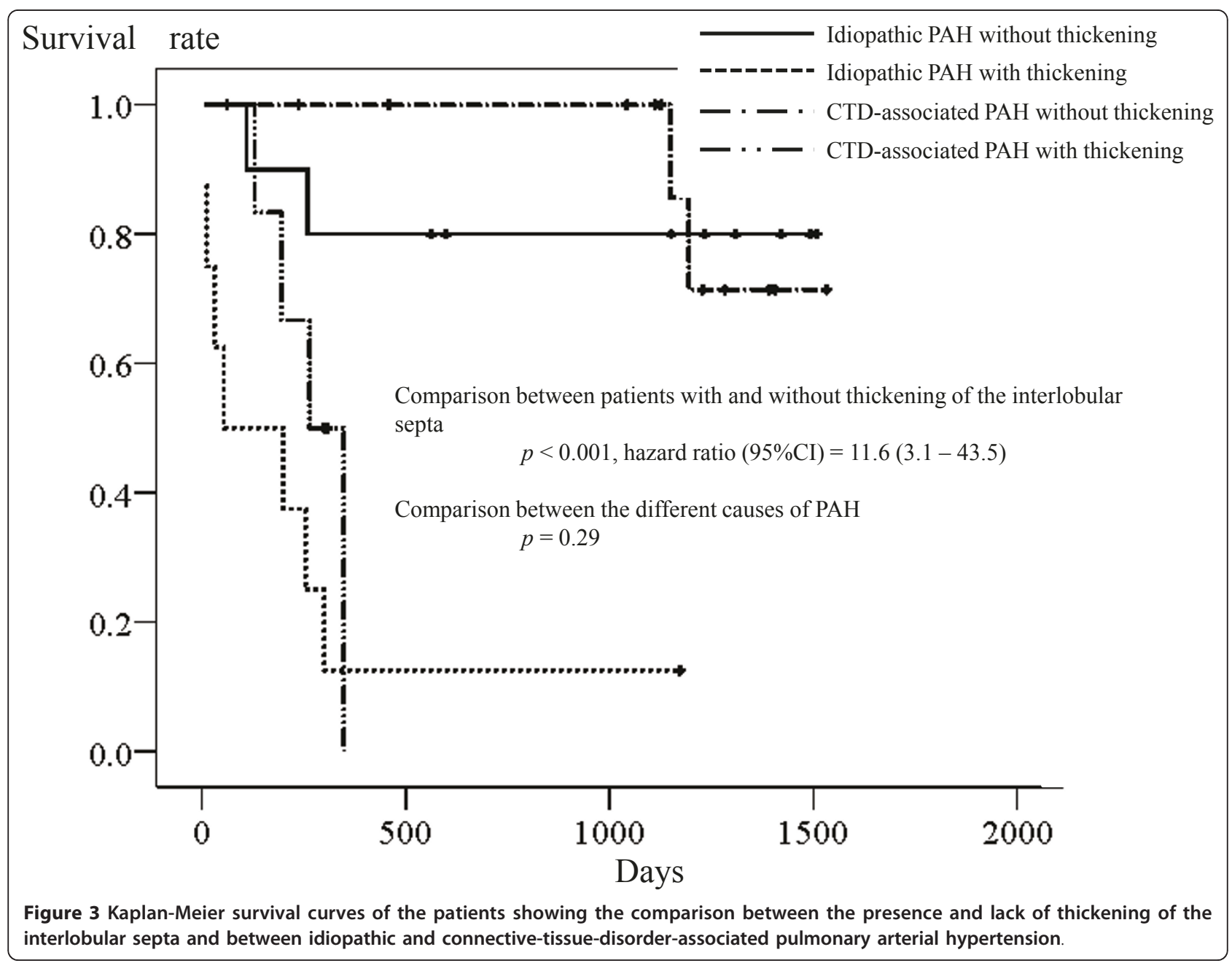


16.9]; BNP: $p<0.001$; hazard ratio $[95 \% \mathrm{CI}]=2.7[1.6-$ 4.5]; the causes of PAH: $p=0.15$ ).

\section{Discussion}

Few studies have assessed obstruction of the pulmonary veins for the risk of death in patients with PAH. The results of the current investigation imply that pulmonary venous obstruction is associated with an elevated risk of death in patients with either idiopathic PAH or CTDassociated PAH. The risk is very large for both causes of PAH. This implication is based on the results of Cox proportional hazard analysis using each radiographic surrogate of pulmonary venous obstruction as follows: mediastinal adenopathy, hazard ratio for death $=13.9(p$ $<0.0001)$; thickening of the interlobular septa, hazard ratio $=12.0(p<0.001)$; and ground-glass attenuation, hazard ratio $=2.0(p=0.02)$.

Recent studies found that obstruction of the pulmonary veins develops in considerable proportion of patients with idiopathic or CTD-associated PAH $[3,4]$. Dorfmüller et al. report that $75 \%$ of patients with CTD- associated and $17 \%$ of patients with non-CTD-associated PAH showed obstruction of the pulmonary veins [3]. Overbeek et al. reported that pulmonary venous obstruction was present in $87.5 \%$ of patients with scleroderma-associated PAH and in $37.5 \%$ of patients with idiopathic PAH [4]. Thus, the differentiation of clinical entities between PAH and PVOD is currently challenged.

For several reasons, obstruction of the pulmonary veins is believed to be related to a malignant prognosis. First, most case reports show that patients with pulmonary venous obstruction die within two years of diagnosis [5-8]; this seems much worse than typical patients with $\mathrm{PAH}[1,2]$. Second, Resten et al. suggest that radiographic surrogates of pulmonary venous obstruction are associated with a poor prognosis [17]. The last report is very important; however, the authors' conclusions were weakened by the study's retrospective design and a lack of survival time analyses [17]. Other than this report, few reports exist examining the effect of pulmonary venous obstruction on the survival of patients with PAH.

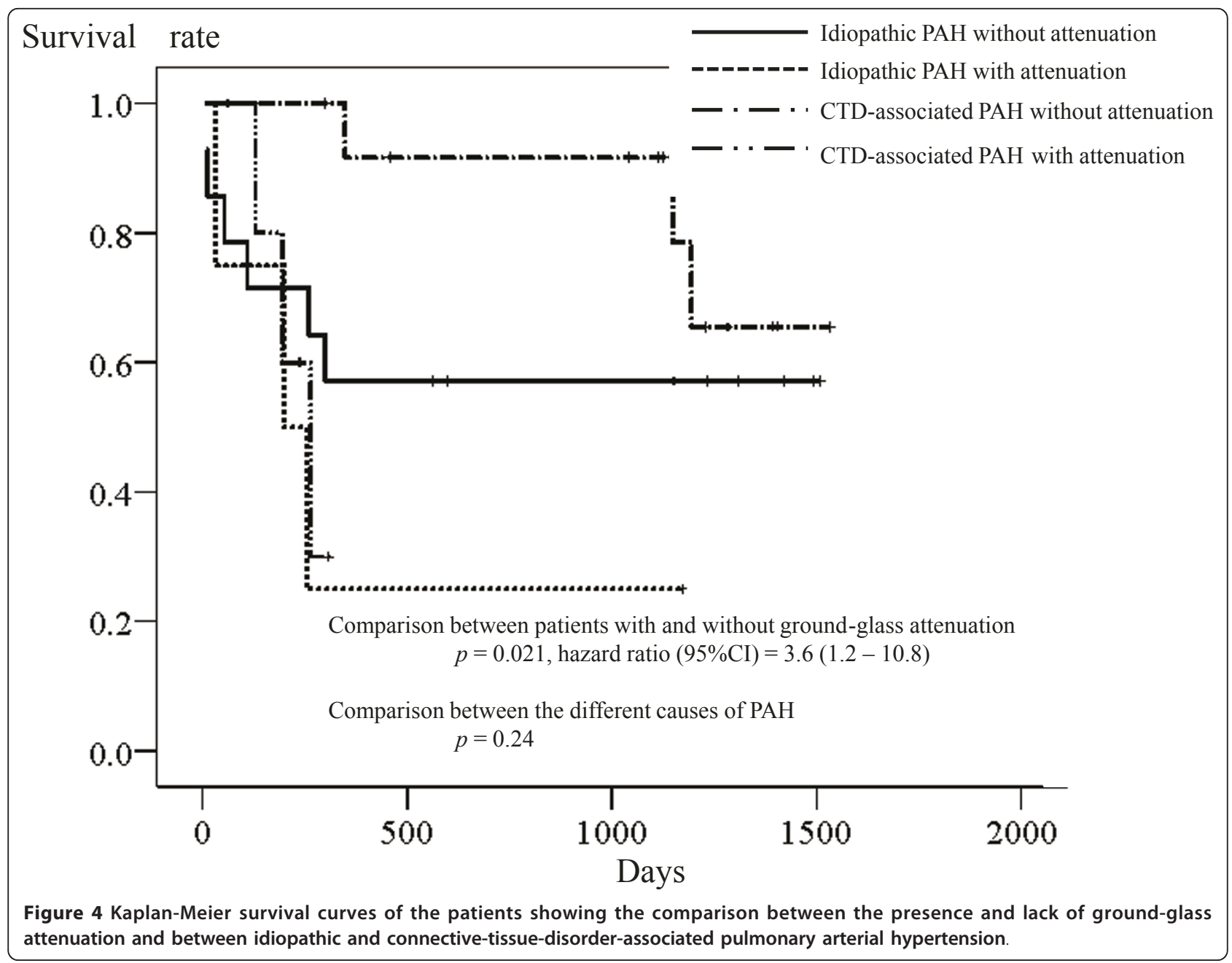


In the current study, radiographic surrogates for the pulmonary venous obstruction had a serious impact on the survival of the patients (Figures 2, 3, and 4). In addition, the surrogates were associated with an increased risk of death independently of the baseline BNP plasma concentration. Therefore, the effect of pulmonary venous obstruction on the mortality rate seemed independent of a poor baseline condition. The survival rate of patients with each surrogate is similar to the reported survival rate of patients with proven pulmonary venous obstruction (as assessed by pathological examinations) [5-8]. Thus, the results of the current study suggest great significance of pulmonary venous obstruction as a mortality determinant.

Studies find that the mortality rate of CTD-associated $\mathrm{PAH}$ is either double or triple that of idiopathic PAH [22-25]. The reason for this discrepancy, however, is unclear. The results of the current study may provide a suggestion of possible cause of this discordance. As described above, one-half or more of patients with CTD-associated PAH have pulmonary venous obstruction, whilst only a minority of patients with idiopathic $\mathrm{PAH}$ do $[3,4]$. In the present study, dividing the patients by the presence or absence of each radiographic surrogate for pulmonary venous obstruction made the survival curves of patients with each cause of PAH close (Figure 2, 3, and 4). These findings suggest, therefore, that the reason for the generally worse prognosis of CTD-associated PAH, compared with idiopathic PAH, is explained by the difference in the frequency of pulmonary venous obstruction at least in part. Given a lack of statistical significance in the difference of mortality rate between the causes of $\mathrm{PAH}$ in this particular patients group, further studies are needed to test this possible explanation for the discordance in prognosis.

Radiographic signs can be used as surrogates as in this study [15]. Studies find that pulmonary radiographic signs on high-resolution CT scan images are successful surrogates for obstruction of the pulmonary veins [15]. A direct diagnosis of pulmonary venous obstruction requires a lung biopsy [11,12]. However, this procedure is hazardous and is available only for select patients $[11,12]$. In addition, guidelines discourage performing a biopsy in frail PAH patients $[11,12]$. Thickening of the interlobular septa, mediastinal adenopathy, and groundglass attenuation are characteristic signs on CT images for obstruction of the pulmonary veins $[11,12,15]$. A previous study demonstrates that the sensitivity and specificity for pulmonary venous obstruction of each radiographic surrogate are as follows: mediastinal adenopathy, $80 \%$ and $100 \%$, respectively; thickening of the interstitial septa, $93 \%$, and $87 \%$, respectively; and the presence of ground-glass attenuation, $87 \%$, and $67 \%$, respectively [15]. Results of two other studies using CT scans agree with these findings $[8,26]$. The results of pathological studies additionally support the rationale for using radiographic surrogates. Pathological studies demonstrate that interstitial pulmonary edema is observed in a few patients without and most patients with obstruction of the pulmonary veins $[3-5,7,8,13,14,26]$. Mediastinal adenopathy is moreover recognised as a frequent and very suggestive radiological feature of pulmonary venous obstruction $[8,15,16,26]$. From these findings, it is apparently possible to use radiographic signs as surrogates for obstruction of the pulmonary veins as in this study. In consideration of the procedural risks, the current study refrained from performing invasive lung biopsies; however, we believe the conclusions of this study are acceptable.

This study had several limitations. This is a single centre investigation; this study therefore has referral biases. We accepted only select patients - the majority of whom had experienced treatment failure at their referring hospitals. This bias was reflected by the poor prognosis of the study patients, the relatively frequent pulmonary venous obstruction among the patients with idiopathic PAH, and a lack of statistical significance in the difference in mortality rate between the patients with idiopathic PAH and those with CTDassociated PAH. Because of the limited number of the patients, the predictive values of each radiographic surrogate were not compared with other characteristics but plasma concentration of BNP. The statistical analyses may have been weak due to the small samples size. Despite of the excellent accuracy, the radiographic surrogates might not completely detect pulmonary venous obstruction.

\section{Conclusions}

In conclusion, the results of this study imply that obstruction of the pulmonary veins is associated with a seriously increased risk of death in patients with PAH.

\section{List of Abbreviations}

BNP: brain natriuretic peptide; CT: computed tomography; CTD: connective tissue disorder; PAH: pulmonary arterial hypertension; PVOD: pulmonary veno-occlusive disease; WHO: World Health Organisation.

\section{Acknowledgements}

The authors thank the following radiologists at our hospital for providing excellent reports of CT images used in this study: Dr. Yuji Shibamoto, Dr. Masaki Hara, Dr. Masato Ito, Dr. Masanori Kitase, Dr. Yuji Mori, Dr. Toshihiro Ito, Dr. Hiromasa Suzuki, Dr. Akihiko Miyamoto, Dr. Hitoshi Tomita, and Dr. Shu-ichi Ohshima.

Funding information

This study was supported by an internal fund of the Nagoya City University Graduate School of Medical Sciences (Nagoya, Japan).

\section{Author details}

${ }^{1}$ Department of Cardio-Renal Medicine and Hypertension, Nagoya City University Graduate School of Medical Sciences, Nagoya, Japan. ${ }^{2}$ Department of Cardiology, Nagoya City Rehabilitation Center, Nagoya, Japan. 


\section{Authors' contributions}

The authors contributed to the study in the following ways: Y. Takeda (Yasuko) participated in the design of the study, obtained the patients' consent, analyzed the reports on CT image, and drafted the manuscript. Yutaka T conceived of the study, participated in its design and coordination, and analyzed the reports on CT image. KY, ST, HN, NO, and GK participated in the design of the study, performed the statistical analysis, and participated to drafting the manuscript. All authors have read and approved the final manuscript.

\section{Competing interests}

The authors declare that they have no competing interests.

Received: 20 November 2010 Accepted: 6 October 2011

Published: 6 October 2011

\section{References}

1. Sitbon $\mathrm{O}$, Humbert M, Nunes H, Parent F, Garcia G, Herve P, Rainisio M, Simonneau G: Long-term intravenous epoprostenol infusion in primary pulmonary hypertension: prognostic factors and survival. J Am Coll Cardiol 2002, 40:780-788.

2. Thenappan T, Shah SJ, Rich S, Gomberg-Maitland M: A USA-based registry for pulmonary arterial hypertension: 1982-2006. Eur Respir J 2007, 30:1103-1110.

3. Dorfmüller P, Humbert M, Perros F, Sanchez O, Simonneau G, Müller KM, Capron F: Fibrous remodeling of the pulmonary venous system in pulmonary arterial hypertension associated with connective tissue diseases. Hum Pathol 2007, 38:893-902.

4. Overbeek MJ, Vonk MC, Boonstra A, Voskuyl AE, Vonk-Noordegraaf A, Smit EF, Dijkmans BA, Postmus PE, Mooi WJ, Heijdra Y, Grünberg K: Pulmonary arterial hypertension in limited cutaneous systemic sclerosis: a distinctive vasculopathy. Eur Respir J 2009, 34:371-379.

5. Mandel J, Mark EJ, Hales CA: Pulmonary veno-occlusive disease. Am J Respir Crit Care Med 2000, 162:1964-1973.

6. Shackelford GD, Sacks EJ, Mullins JD, McAlister WH: Pulmonary venoocclusive disease: case report and review of the literature. AJR Am J Roentgenol 1977, 128:643-648.

7. Montani D, Achouh L, Dorfmüller P, Le Pavec J, Sztrymf B, Tchérakian C, Rabiller A, Haque R, Sitbon O, Jaïs X, Dartevelle P, Maitre S, Capron F, Musset $D$, Simonneau G, Humbert M: Pulmonary veno-occlusive disease: clinical, functional, radiologic, and hemodynamic characteristics and outcome of 24 cases confirmed by histology. Medicine (Baltimore) 2008, 87:220-233.

8. Holcomb BW Jr, Loyd JE, Ely EW, Johnson J, Robbins IM: Pulmonary venoocclusive disease: a case series and new observations. Chest 2000, 118:1671-1679.

9. McGoon M, Gutterman D, Steen V, Barst R, McCrory DC, Fortin TA, Loyd JE: Screening, early detection, and diagnosis of pulmonary arterial hypertension: ACCP evidence-based clinical practice guidelines. Chest 2004, 126:14S-34S

10. Simonneau G, Galie N, Rubin L, Langleben D, Seeger W, Domenighetti G, Gibbs S, Lebrec D, Speich R, Beghetti M, Rich S, Fishman A: Clinical classification of pulmonary hypertension. J Am Coll Cardiol 2004, 43:5S-12S.

11. Galiè N, Hoeper MM, Humbert M, Torbicki A, Vachiery JL, Barbera JA, Beghetti M, Corris P, Gaine S, Gibbs JS, Gomez-Sanchez MA, Jondeau G, Klepetko W, Opitz C, Peacock A, Rubin L, Zellweger M, Simonneau G, ESC Committee for Practice Guidelines (CPG), Vahanian A, Auricchio A, Bax J, Ceconi C, Dean V, Filippatos G, Funck-Brentano C, Hobbs R, Kearney P, McDonagh T, McGregor K, Popescu BA, Reiner Z, Sechtem U, Sirnes PA, Tendera M, Vardas P, Widimsky P, Document Reviewers, Sechtem U, Al Attar N, Andreotti F, Aschermann M, Asteggiano R, Benza R, Berger R, Bonnet D, Delcroix M, Howard L, Kitsiou AN, Lang I, Maggioni A, NielsenKudsk JE, Park M, Perrone-Filardi P, Price S, Domenech MT, VonkNoordegraaf A, Zamorano JL: Guidelines for the diagnosis and treatment of pulmonary hypertension: The Task Force for the Diagnosis and Treatment of Pulmonary Hypertension of the European Society of Cardiology (ESC) and the European Respiratory Society (ERS), endorsed by the International Society of Heart and Lung Transplantation (ISHLT). Eur Heart J 2009, 30:2493-2537.
12. McLaughlin W, Archer SL, Badesch DB, Barst RJ, Farber HW, Lindner JR, Mathier MA, McGoon MD, Park MH, Rosenson RS, Rubin L, Tapson VF, Varga J: ACCF/AHA 2009 expert consensus document on pulmonary hypertension a report of the American College of Cardiology Foundation Task Force on Expert Consensus Documents and the American Heart Association developed in collaboration with the American College of Chest Physicians; American Thoracic Society, Inc.; and the Pulmonary Hypertension Association. J Am Coll Cardiol 2009, 53:1573-1619.

13. Montani D, Kemp K, Dorfmuller P, Sitbon O, Simonneau G, Humbert M: Idiopathic pulmonary arterial hypertension and pulmonary venoocclusive disease: similarities and differences. Semin Respir Crit Care Med 2009, 30:411-420.

14. Rabiller A, Jaïs X, Hamid A, Resten A, Parent F, Haque R, Capron F, Sitbon O, Simonneau G, Humbert M: Occult alveolar haemorrhage in pulmonary veno-occlusive disease. Eur Respir J 2006, 27:108-113.

15. Resten A, Maitre S, Humbert M, Rabiller A, Sitbon O, Capron F, Simonneau G, Musset D: Pulmonary hypertension: $\mathrm{CT}$ of the chest in pulmonary venoocclusive disease. AJR Am J Roentgenol 2004, 183:65-70.

16. Thomas de Montpréville V, Dulmet E, Fadel E, Dartevelle P: Lymph node pathology in pulmonary veno-occlusive disease and pulmonary capillary heamangiomatosis. Virchows Arch 2008, 453:171-176.

17. Resten A, Maitre S, Humbert M, Sitbon O, Capron F, Simoneau G, Musset D: Pulmonary arterial hypertension: thin-section CT predictors of epoprostenol therapy failure. Radiology 2002, 222:782-788.

18. Webb WR, Stein MG, Finkbeiner WE, Im JG, Lynch D, Gamsu G: Normal and diseased isolated lungs: high-resolution CT. Radiology 1988, 166:81-87.

19. Engeler CE, Tashjian JH, Trenkner SW, Walsh JW: Ground-glass opacity of the lung parenchyma: a guide to analysis with high-resolution CT. AJR Am J Roentgenol 1993, 160:249-251.

20. Richards KL, Cannon SR, Miller JF, Crawford MH: Calculation of aortic valve area by Doppler echocardiography: a direct application of the continuity equation. Circulation 1986, 73:964-969.

21. Takeda Y, Takeda Y, Suzuki S, Kimura G: Within-person variation of the plasma concentration of B-type natriuretic peptide: safety range in stable patients with heart failure. Am Heart J 2009, 157:97-101.

22. Kuhn KP, Byrne DW, Arbogast PG, Doyle TP, Loyd JE, Robbins IM: Outcome in 91 consecutive patients with pulmonary arterial hypertension receiving epoprostenol. Am J Respir Crit Care Med 2003, 167:580-586.

23. Kawut SM, Taichman DB, Archer-Chicko CL, Palevsky HI, Kimmel SE: Hemodynamics and survival in patients with pulmonary arterial hypertension related to systemic sclerosis. Chest 2003, 123:344-350.

24. Ruiz-Cano MJ, Escribano P, Alonso R, Delgado J, Carreira P, Velazquez T, Sanchez MA, Sáenz de la Calzada C: Comparison of baseline characteristics and survival between patients with idiopathic and connective tissue disease-related pulmonary arterial hypertension. $J$ Heart Lung Transplant 2009, 28:621-627.

25. Chung L, Liu J, Parsons L, Hassoun PM, McGoon M, Badesch D, Miller DP, Nicolls MR, Zamanian RT: Characterisation of connective tissue disease associated pulmonary arterial hypertension from the REVEAL Registry: identifying systemic sclerosis as a unique phenotype [published ahead of print]. Chest 2010.

26. Montani $D$, Jaïs $X$, Price $L C$, Achouh $L$, Degano B, Mercier O, Mussot $S$, Fadel E, Dartevelle P, Sitbon O, Simonneau G, Humbert M: Cautious epoprostenol therapy is a safe bridge to lung transplantation in pulmonary veno-occlusive disease. Eur Respir J 2009, 34:1348-1356.

\section{Pre-publication history}

The pre-publication history for this paper can be accessed here: http://www.biomedcentral.com/1471-2466/11/47/prepub

doi:10.1186/1471-2466-11-47

Cite this article as: Takeda et al:: Pulmonary venous occlusion and death in pulmonary arterial hypertension: survival analyses using radiographic surrogates. BMC Pulmonary Medicine 2011 11:47. 